\title{
Individual coactivation patterns improve the subject identification and their behavior association
}

Hang Yang ${ }^{1}$, Xing Yao ${ }^{1}$, Hong Zhang ${ }^{1}$, Chun Meng ${ }^{1}$, Bharat Biswal ${ }^{1,2^{*}}$

${ }^{1}$ The Clinical Hospital of Chengdu Brain Science Institute, MOE Key Laboratory for Neuroinformation, Center for Information in Medicine, School of Life Science and Technology, University of Electronic Science and Technology of China, Chengdu 611731, China.

${ }^{2}$ Department of Biomedical Engineering, New Jersey Institute of Technology, Newark, NJ, 07102, USA.

\section{* Correspondence:}

Dr. Bharat B. Biswal, 607 Fenster Hall, University Height, Newark, NJ, 07102,

USA; E-mail: bbiswal@gmail.com 


\section{Abstract}

Brain states can be characterized by recurring coactivation patterns (CAPs).

Traditional CAP analysis is performed at the group-level, while the human brain is individualized and the functional connectome has shown the uniqueness as fingerprints.

Whether stable individual CAPs could be obtained from a single fMRI scan and could individual CAPs improve the identification is unclear. An open dataset, the midnight scan club was used in this study to answer these questions. Four CAP states were identified at three distinct levels (group-, subject- and scan-level) separately, and the CAPs were then reconstructed for each scan. Identification rate and differential identifiability were used to evaluate the subject identification performance. Our results demonstrated that the individual CAPs were unstable when using a single scan. By maintaining high intra-subject similarity and inter-subject differences, subject-level CAPs achieved the best identification performance. Particularly, brain regions that contributed to the identifiability were mainly located in higher-order networks (e.g., frontal-parietal network). Besides, head motion reduced the intra-subject similarity, while its impact on identification rate was non-significant. Finally, a pipeline was developed to depict brain-behavior associations in datasets with few samples and dense sampling, and individualized CAP dynamics showed an above-chance level correlation with IQ.

Keywords: coactivation patterns, individual analysis, subject identification, differential identifiability 


\section{Introduction}

Brain dynamics has been a topic of great interest in the last decade (Hutchison et al. 2013; Preti et al. 2017). One important question is identifying dynamic brain states characterized by specific spatial and temporal profiles (Allen et al. 2014; Vidaurre et al. 2017). The temporally-varied interactions between brain regions and networks are generally measured by dynamic functional connectivity (dFC), and FC-states can be further estimated by identifying recurring connectivity patterns across sliding windows (Allen et al. 2014). Recently, coactivation patterns (CAPs), a volume-based method that can capture brain dynamics at a higher temporal resolution than the sliding-window approach, has been developed (Liu and Duyn 2013; Liu et al. 2018). The CAP method can be performed in a seed-based (Chen et al. 2015; Liu and Duyn 2013) or seed-free manner (Liu et al. 2013). It has been applied in studies about cognitive flexibility (Kupis et al. 2021), consciousness (Huang et al. 2020), gender differences (Murray et al. 2021), frequency-specific dynamics (Yang et al. 2021a) and disease alterations (Kaiser et al. 2019; Piguet et al. 2021; Rey et al. 2021). Besides, the robustness and repeatability of CAPs have been validated across various analytic pipelines and independent datasets (Yang et al. 2021b), and have been further improved by using multiband multi-echo imaging (Cohen et al. 2021).

Group-level fMRI studies mainly focus on the consistent patterns across individuals, and have established essential knowledge about the brain structure and functional organization (Buckner et al. 2013; Glasser et al. 2016), cognition (Cole et al. 2014), and clinical populations (Fornito et al. 2015). However, individual brains are not 
only heterogeneous in psychiatric populations (Marquand et al. 2016; Price et al. 2017), but also varied in healthy controls (Mueller et al. 2013). Recent studies have started shifting from group-level analyses to individual studies (Dubois and Adolphs 2016), with several studies trying to customize a personalized functional topography for each individual (Gordon et al. 2017a; Wang et al. 2015). The reliability of individual brain features has been validated over time (Gratton et al. 2018; Horien et al. 2019), between datasets (Gordon et al. 2017a) and tasks (Laumann et al. 2015). Besides, the validity of individual brain features has also been proved in several areas, including youth development (Cui et al. 2020), brain-behavior prediction (Kong et al. 2019; Sui et al. 2020; Wang et al. 2018), clinical intervention (Cash et al. 2021; Lynch et al. 2019) and treatment prediction (Wang et al. 2020). These studies have shown the significance of individual variations and shed light on precision psychiatry in the future (Gratton et al. 2020; Sylvester et al. 2020).

Among these studies focusing on individual subjects, one important question is recognizing reliable brain features that can stably identify an individual from a pool of people. By matching the connectivity profile of one session with other sessions, Finn and colleagues found the individual functional connectome is unique and could serve as the fingerprint for subject identification (Finn et al. 2015). Several studies since then have further validated the reliability and validity of connectome fingerprints (Finn et al. 2017; Kaufmann et al. 2017; Pannunzi et al. 2017; Sorrentino et al. 2021). Besides the functional connectivity, effective connectivity from task fMRI (Pallares et al. 2018) and white matter fibers (Kumar et al. 2017) are also unique for each individual and able to 
distinguish between subjects. Most of the current subject identification studies are based on static functional connectivity, while the brain dynamics of each individual are also meaningful features and could provide additional information to identifiability (Menon and Krishnamurthy 2019). Coactivation patterns represent dynamic brain states characterized by specific coactivation profiles, while current CAP studies are mainly performed at the group-level by using data from all participants, it is unclear whether individual CAPs are stable. In addition, could distinct CAPs identify individuals from each other, and whether individualized CAPs would further improve the subject identification accuracy are remained to be answered.

In this study, the midnight scan club (MSC) dataset was used to study whether individual CAP states can be stably identified by using a single scan, and whether individual CAPs could enlarge the inter-subject differences and increase the subject identification accuracy. We first generated CAP states at three levels, including the group-level, subject-level and scan-level. Then, the subject identification ability was assessed by using both differential identifiability and identification (ID) rate, and the contribution of brain regions has also been estimated. Besides, the impacts of head movement on CAPs and subject identification were evaluated. Finally, we developed a random-based framework to build the brain-behavior relationship in the precision dataset with few subjects but repeated scans.

\section{Materials and Methods}

\subsection{Dataset and preprocessing}


The publicly available midnight scan club (MSC) dataset, which includes data from ten adults $(5$ females, age $=29.1 \pm 3.3)$ was used in this work (Gordon et al. 2017b). Ten resting-state fMRI scans from each subject were collected on separate days, using the gradient-echo EPI sequence $\left(\mathrm{TR}=2.2 \mathrm{~s}, \mathrm{TE}=27 \mathrm{~ms}\right.$, flip angle $=90^{\circ}$, voxel size $=$ $4 \times 4 \times 4 \mathrm{~mm}^{3}, 36$ slices) on a Siemens TRIO 3T MRI scanner. Each scan lasts for 30 minutes (818 volumes) and begins at midnight. Subject MSC08 was excluded in this work as the subject fell asleep during the scan, hence nine subjects with a total of ninety scans were analyzed. More detailed information about the participant information and MRI acquisition parameters can be found in (Gordon et al. 2017b). Particularly, Kaufman brief intelligence test, second edition (KBIT2) was used to evaluate participants' IQ (Kaufman 2004).

$\begin{array}{llll}\text { Preprocessed } & \text { fromages the OpenfMRI }\end{array}$ (https://openneuro.org/datasets/ds000224) were used in the current work. In brief, it includes slice timing correction, intensity normalization (mode $=1,000)$, within-subject head motion correction, and distortion correction. The functional data were registered to Talairach atlas space using the average T2-weighted image and the average T1weighted image. To account for anatomical differences between subjects, we used the FSL's FNIRT to non-linearly warp the mean T1 image to the MNI space, and the preprocessed volumetric time series at Talairach space were then registered to the registered T1 image at the MNI space (Greene et al. 2020). Schaefer's 400 cortical regions (Schaefer et al. 2018), which include the visual network (VN), somatomotor network (SMN), dorsal attention network (DAN), ventral attention network (VAN), 
limbic network, fronto-parietal network (FPN) and default mode network (DMN) (Yeo et al. 2011), were used to extract the ROI-level BOLD signal. Before the CAP analysis, the time series from each ROI were normalized using z-score separately for each scan.

\subsection{Coactivation pattern analysis}

The brain states can be characterized by specific whole-brain coactivation patterns, using methods, e.g., k-means clustering (Liu et al. 2013; Liu and Duyn 2013). Our previous study has developed a robust CAP analysis pipeline (Yang et al. 2021b). In this work, we performed the CAP analysis pipeline at three levels, including the grouplevel, subject-level and scan-level (Figure 1A). As for the group-level, all the 90 scans were cascaded, which groups as a $(73,620 * 400)$ coactivation matrix, where 73,620 $\left(818^{*} 90\right)$ is the total volumes and 400 is the ROI number. The between-volume distance was calculated by using correlation distance ( 1 - Pearson correlation coefficient), and k-means clustering was then performed to identify brain states with similar coactivation profiles. The group-level CAP maps were obtained by averaging volumes assigned to the same state, and divided by the within-cluster standard deviation. We tested cluster number $\mathrm{K}$ from 2 to 21 with step length $=1$. For the subject-level CAPs, each subject's ten scans were grouped as an $(8,180 * 400)$ coactivation matrix, and a similar clustering procedure was performed for each subject independently. Finally, the scan-level CAPs were generated by applying the CAP pipeline to the $(818 * 400)$ coactivation matrix from each scan. Based on the elbow method (Supplementary Figure S1), K = 4 was used for the three levels in our further analysis. 
To match and compare the CAPs obtained from the three levels, the group-level CAP maps were used as references, the subject-level and scan-level CAPs were then aligned by using the Hungarian algorithm (Gutierrez-Barragan et al. 2019; Kuhn 1955; Tarun et al. 2020). In addition, for the group-level and subject-level results, CAP maps of each scan were also reconstructed by averaging the volumes belonging to every single scan. Therefore, there were four CAP states of each level per scan, and all the following analyses were performed based on these reconstructed CAPs. In the current study, we mainly calculated three primary CAP matrices to measure the temporal dynamics of brain states. Fraction of time is defined as the percentage of volumes assigned to one state, and persistence measures the average duration time of one state before it changes to another state, counts records the occurrence frequency of one state during the whole scan.

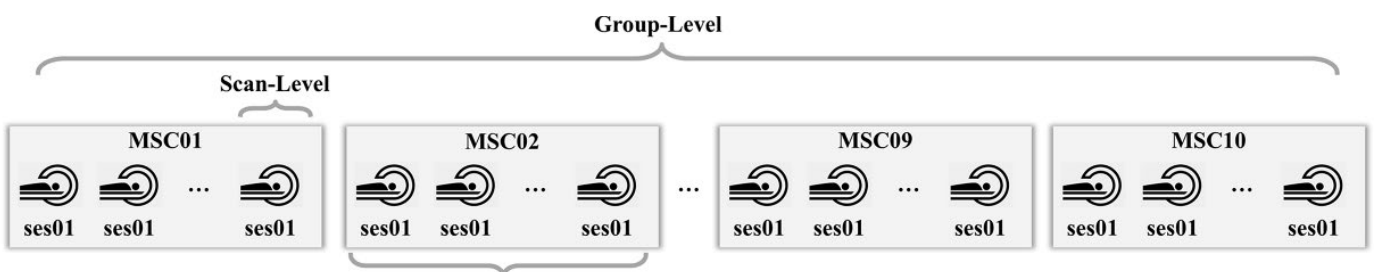

A
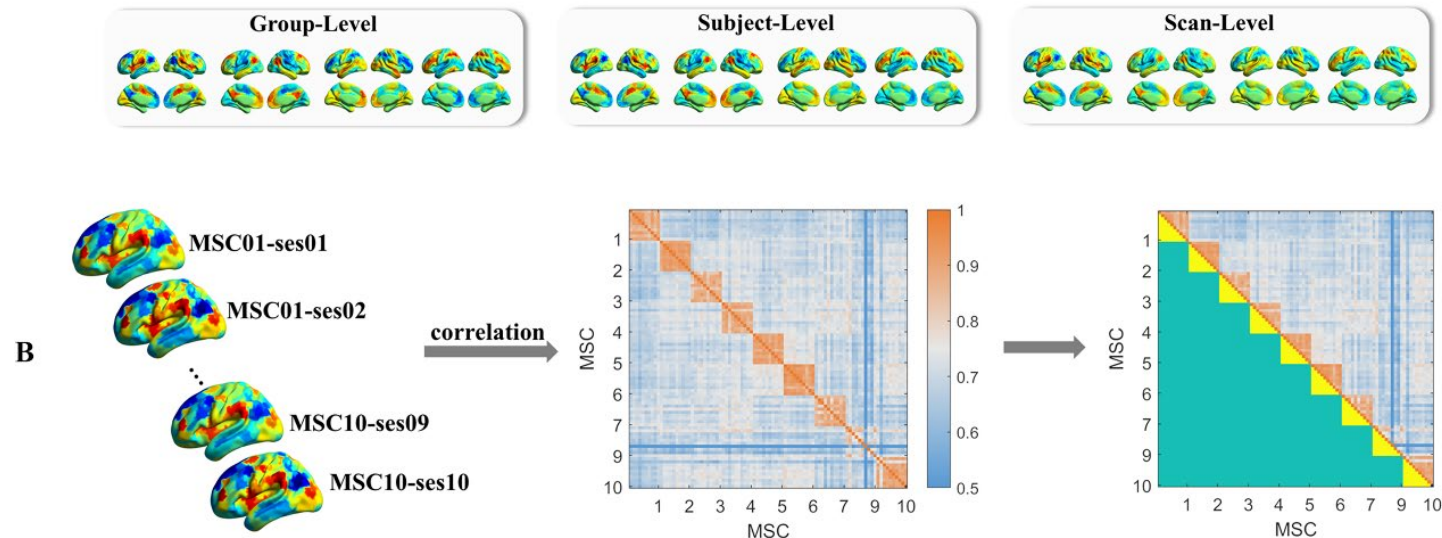

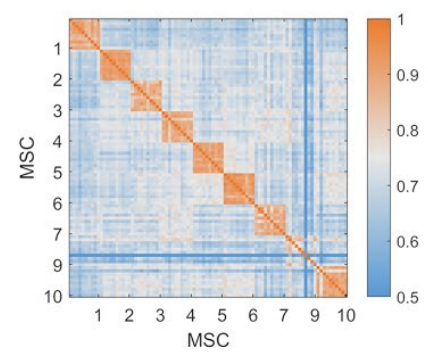

identifiability matrix

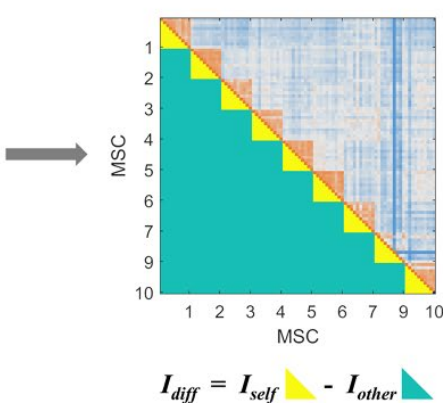

Figure 1. A) The CAP pipeline performed at the three levels. The group-level CAPs were obtained using all subjects' scans, the subject-level CAPs were generated based 
on all scans from each single subject, and the scan-level CAPs were acquired by using the single scan. B) Spatial similarity between all CAP maps from all scans was measured by Pearson correlation, and the correlation matrix was called identifiability matrix. Differential identifiability $\left(\boldsymbol{I}_{\text {diff }}\right)$ was calculated by subtracting the average intersubject similarity $\left(\boldsymbol{I}_{\text {other }}\right)$ from the average intra-subject similarity $\left(\boldsymbol{I}_{\text {self }}\right)$.

\subsection{Subject identification and differential identifiability}

Recently, Finn and colleagues have shown the functional connectome as a robust fingerprint (Finn et al. 2015). By calculating the Pearson correlation between the sample FC from Day 1 and all FC from Day 2, the other scans from the sample subject showed a stronger correlation compared with scans from different subjects. In our work, a similar subject identification procedure was performed. First, Pearson correlation was calculated between CAP maps of the ninety scans to evaluate the similarity across scans and subjects, termed as identifiability matrix (Figure $1 \mathrm{~b}$ ). Then, for each scan, we tested whether it could find the other nine scans from the same subject by choosing scans with the top nine Pearson correlation coefficients. The accuracy of each scan was defined as the percentage of correct identifications, defined as the identification (ID) rate. The CAP variance maps were also generated from the ninety scans, and brain region with larger variance is supposed to be more important to distinguish between individuals.

Besides the target-based subject identification method, another quantification metric called differential identifiability $\left(\boldsymbol{I}_{\text {diff }}\right)$ was also used in this study (Amico and 
Goni 2018). The $\boldsymbol{I}_{\text {diff }}$ is defined as the differences between the average intra-subject similarity $\left(\boldsymbol{I}_{\text {self }}\right.$, diagonal of the identifiability matrix $)$ and inter-subject similarity $\left(\boldsymbol{I}_{\text {other }}\right.$, off-diagonal of the identifiability matrix). A larger $\boldsymbol{I}_{\text {diff }}$ represents better identifiability. All values $\left(\boldsymbol{I}_{\text {self }}\right.$, $\left.\boldsymbol{I}_{\text {other }}, \boldsymbol{I}_{\text {diff }}\right)$ were multiplied by 100 for a more straightforward demonstration. To account for the contribution of region $\boldsymbol{i}$, the identifiability matrix and differential identifiability was re-evaluated by removing the $\boldsymbol{R} \boldsymbol{O} \boldsymbol{I}_{\boldsymbol{i}}$, and the changed differential identifiability $\left(\boldsymbol{\Delta} \boldsymbol{I}_{\text {diff }}\right)$ was calculated by subtracting the original $\boldsymbol{I}_{\text {diff }}$ from the new $\boldsymbol{I}_{\text {diff. }}$. A positive $-\boldsymbol{\Delta} \boldsymbol{I}_{\text {diff }}$ indicates a positive contribution of the $\boldsymbol{R} \boldsymbol{O} \boldsymbol{I}_{i}$, as a negative $\boldsymbol{\Delta} \boldsymbol{I}_{\text {diff }}$ means the new $\boldsymbol{I}_{\text {diff }}$ decreased when removing the $\boldsymbol{R} \boldsymbol{O} \boldsymbol{I}_{\boldsymbol{i}}$. The contribution of each functional network was estimated in a similar manner, by removing all regions from the network.

\subsection{Effects of head movement}

In this study, the effects of head movement on CAP results and subject identification were evaluated. First, framewise displacement (FD) was calculated to measure the head movement (Power et al. 2012). The temporal mask of each scan was created by labeling frames with FD $>0.2 \mathrm{~mm}$ as motion-contaminated, and the number of motion-contaminated frames was counted to represent its head movement level. Scans with more motion-contaminated frames are supposed to be less similar to the other scans from the same subject. Therefore, the spatial similarity of scan $\boldsymbol{i}$ from subject $\boldsymbol{j}$ to the other nine scans from the same subject was averaged and defined as $\boldsymbol{I}_{\text {self }}$ $(\boldsymbol{i}, \boldsymbol{j})$. Then, Spearman correlation was calculated between the head movement level and 
$\boldsymbol{I}_{\text {self }}(\boldsymbol{i}, \boldsymbol{j})$ across the ninety scans. In addition, the ninety scans were ordered based on the number of motion-contaminated frames, and the identification rate of the top 30 scans and the bottom 30 scans were compared by using permutation test (5,000 times).

\subsection{Brain-behavior correlation}

To the best of our knowledge, no study has analyzed the brain-behavior relationship in the MSC dataset. The challenge is that, there are ten scans and ten corresponding brain measures for each subject, while there is only one value for each type of behavior scale. Considering the minimal number of subjects, it is difficult to build a statistically meaningful model to capture a robust brain-behavior association.

To solve this problem, we used a randomization strategy to create the augmented data. The basic assumption is that, if there exists a stable brain-behavior association, then brain measures obtained from any session should also work. As shown in Figure 2 , the original data group $\mathbf{X}(10 * 9)$ consisted of the data from the nine subjects acquired at ten sessions. To build the augmented data group $\mathbf{Y}$, the session order from each subject (the row of $\mathbf{X}$ ) was randomized independently, then each column of $\mathbf{Y}$ included the data of the nine subjects from different sessions. Finally, the random data group $\mathbf{Z}$ was constructed by shuffling the column of $\mathbf{Y}$, and the correspondence between brain measures and behavior measures was disrupted. The randomization process was repeated 1,000 times, which generated 10,000 augmented sub-groups and 10,000 shuffled sub-groups. Spearman correlation was calculated between CAP matrices and behavior measures for each sub-group, which generates a true distribution (for 
augmented data) and a null distribution (for shuffled data). Two-sample t-test was used to test the differences between the two distributions, and the Cohen-d value was calculated to measure the effect size. For a simple demonstration, we only used the total IQ score (KBIT-2) in this work.

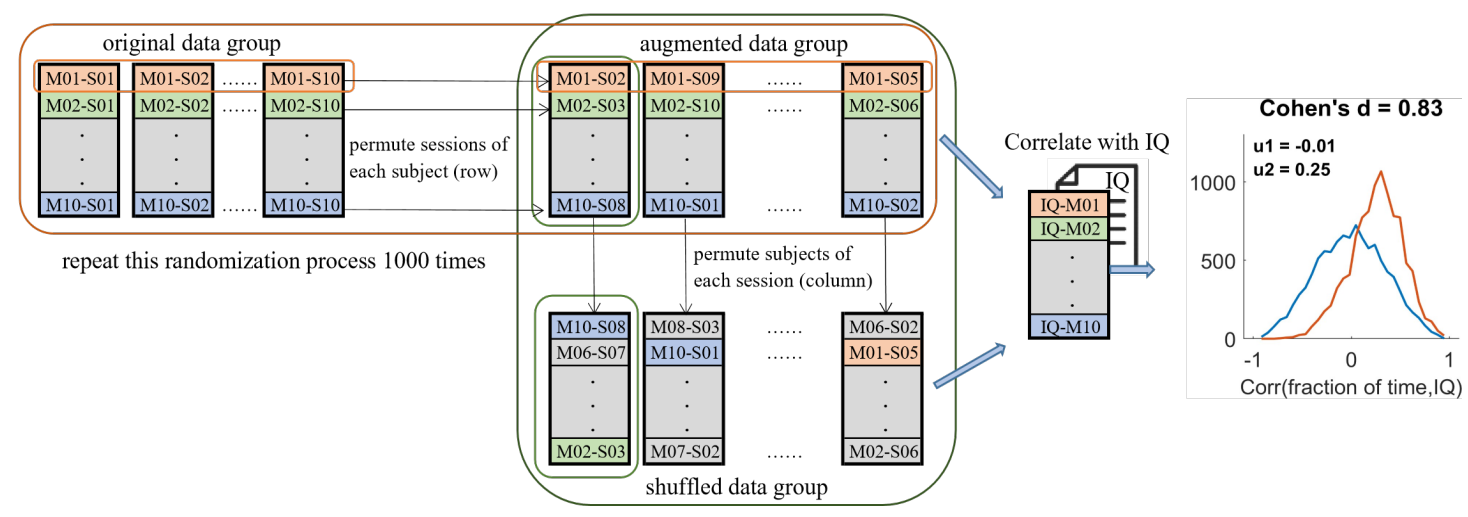

Figure 2. The pipeline of random-based brain-behavior correlation analysis in a dataset with few subjects but multiple scans. The original data group includes the data from the same session, e.g., M01-S01 means session 1 of subject MSC01. The augmented data group was generated by permuting sessions of each subject, and the shuffled data group was obtained by further permuting subjects of each session. Then, Spearman correlations were calculated between brain measures from the augmented/shuffled data group and IQ independently.

\section{Results}

\subsection{CAPs generated at distinct levels}

Four CAP states were identified and analyzed in this study at the three levels, and CAP maps at the subject-level and scan-level were matched with the group-level. Group-representative CAP maps at the three levels are shown in Figure 3, and two CAP 
pairs were obtained with opposite coactivation profiles. The relative activation level of each functional network is shown in the supplementary Figure S2. It can be observed that State 1 was mainly dominated by the activated VAN, SMN and de-activated DMN, and the opposite pattern was observed in State 2. State 3 showed stronger de-activation in the FPN, DAN and activation in the anterior DMN, and the opposite for State 4.

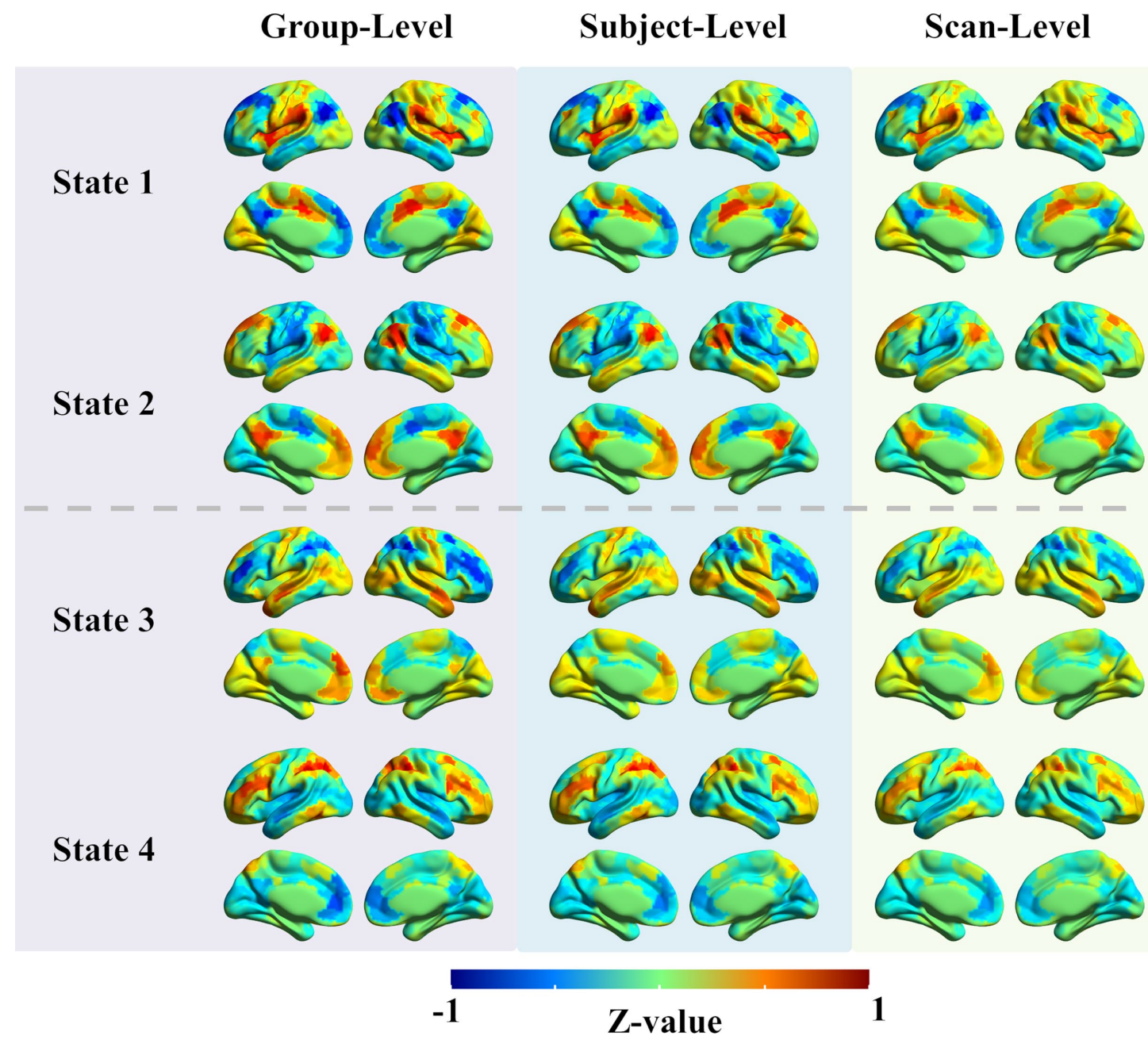

Figure 3. The CAP maps at the group-level, subject-level and scan-level. Four CAPs were analyzed in this work, and two CAP pairs with opposite spatial patterns were identified. State 1 and 2 were mainly dominated by stronger activation or de-activation in the DMN, VAN and SMN. State 3 and 4 were primarily dominated by stronger activation or de-activation in the FPN, DAN and anterior DMN. The red color indicates 
a stronger activation level than the baseline, and vice versa for the blue color.

Abbreviations: DAN, dorsal attention network; DMN, default mode network; FPN,

fronto-parietal network; SMN, somatomotor network; VAN, ventral attention network.

\subsection{Subject identification and differential identifiability}

First, the identifiability matrix was calculated at the three levels separately. As shown in Figure 4A, two opposite CAP pairs can be observed, where the first 90 scans came from State 1 and the following 90 scans came from State 2, and similar patterns for the last 180 scans for State 3 and 4. It can also be observed that within each state, scans from the same subject were more similar than scans from other subjects. The identifiability matrices of State 1 at the three levels were further extracted as examples (Figure 4B). Both the group-level and subject-level showed high intra-subject similarity, while only MSC02 and MSC06 showed consistent high intra-subject similarity across the ten scans at the scan-level. Besides, a lower inter-subject similarity can be observed at the subject-level than group-level.

Group-Level
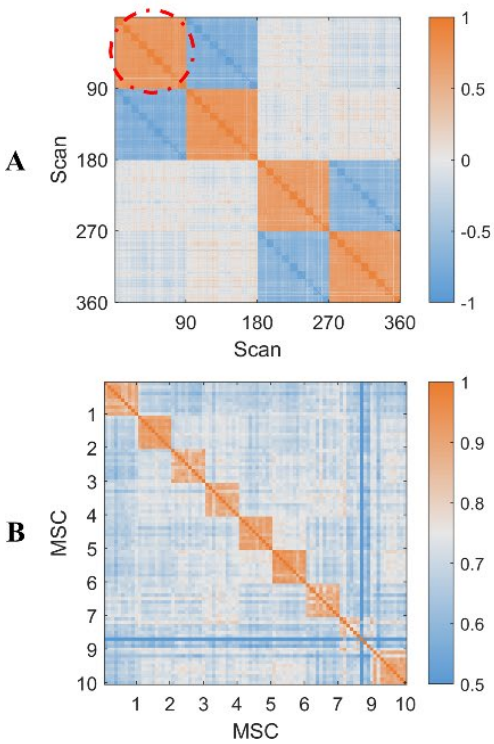

Subject-Level
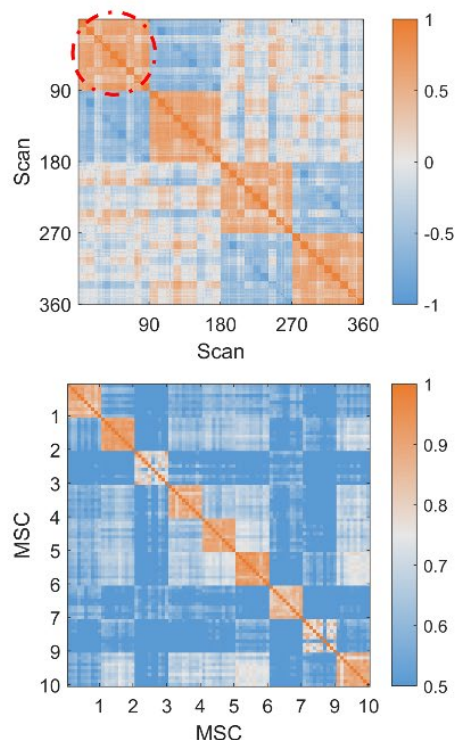

Scan-Level
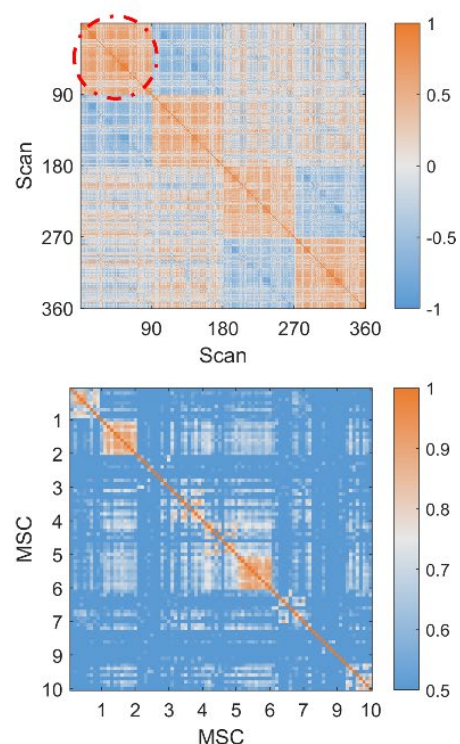
Figure 4. Identifiability matrix at the three levels. A) Identifiability matrix of the four CAP states. Scan 1-90, 91-180, 181-270 and 271-360 came from State 1 to State 4 separately. B) Identifiability matrix of State 1. The colorbar shows the Pearson correlation coefficient.

The scan-to-scan identification accuracy and differential identifiability were calculated based on the identifiability matrix from each state separately. As shown in Figure 5A, group-level showed high intra-subject similarity $\left(\boldsymbol{I}_{\text {self }}>80\right)$ and inter-subject similarity $\left(\boldsymbol{I}_{\boldsymbol{o t h e r}}>60\right)$ across the four CAP states. On the contrary, scan-level CAPs were characterized by small $\boldsymbol{I}_{\text {self }}(<60)$ and $\boldsymbol{I}_{\text {other }}(<40)$. Hence, group-level and scanlevel showed comparable low differential identifiability $\left(\boldsymbol{I}_{\text {diff }}<20\right)$. However, grouplevel showed a high ID rate (>90\%), but scan-level achieved less than $50 \%$ accuracy. As for the subject-level CAPs, while maintaining high intra-subject similarity, the intersubject similarity was also reduced, which leads to the highest differential identifiability $\left(\boldsymbol{I}_{\text {diff }}=32.96,28.63,36.77,33.78\right.$, from State 1 to State 4$)$ among the three levels. Besides, subject-level also showed the highest scan-to-scan ID rate. The detailed values of the differential identifiability and identification rate can be found in the supplementary Table S1, S2. 
A
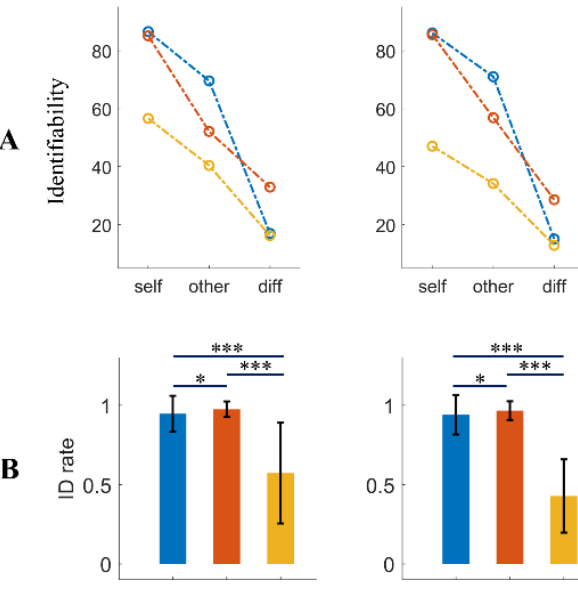

State 1

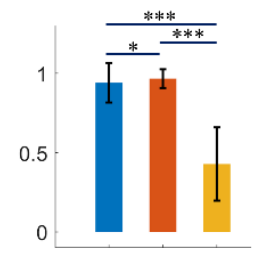

State 2
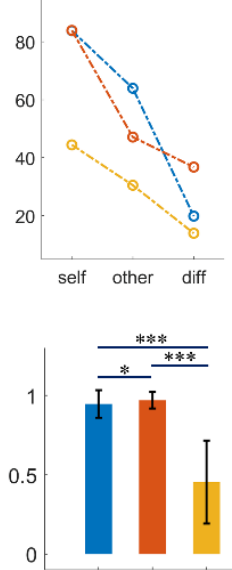

State 3

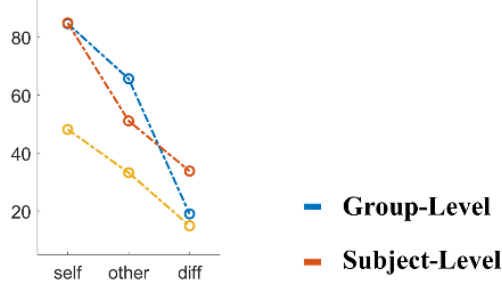

- Scan-Level

Figure 5. Subject identification ability at the three levels. A) The intra-subject similarity $\left(\boldsymbol{I}_{\text {self }}\right)$, inter-subject similarity $\left(\boldsymbol{I}_{\text {other }}\right)$, and differential identifiability $\left(\boldsymbol{I}_{\text {diff }}\right)$. B) The scanto-scan identification (ID) rate. Permutation test was performed between each pair of levels, and false discovery rate (FDR) was used to correct multiple comparisons. * indicates $\mathrm{P}<0.05$, and $* * *$ indicates $\mathrm{P}<0.0001$. Errorbar indicates the standard deviation.

To further investigate the potential contribution of each brain region to the subject identification, variance maps were first calculated across the ninety scans for each CAP state separately. In the main text, we mainly reported results at the subject-level. Very similar variance patterns were found between the four CAP states. Brain regions with larger variances were mainly located at the left hemisphere, including the middle frontal gyrus, superior parietal lobe and supramarginal gyrus (Figure 6A). Similar variance distribution was also found at the group-level and scan-level, and scan-level showed overall larger variances across the whole brain (supplementary Figure S3, S4). In addition, we also measured the changed differential identifiability $\left(\Delta \boldsymbol{I}_{\text {diff }}\right)$ of each brain 
region, and the $-\boldsymbol{\Delta} \boldsymbol{I}_{\text {diff }}$ was reported as a positive $-\boldsymbol{\Delta} \boldsymbol{I}_{\text {diff }}$ indicates a positive contribution ( $\boldsymbol{I}_{\text {diff }}$ decreased when excluding the specific region). Unlike the variance maps that were very similar across CAP states, $-\boldsymbol{\Delta} \boldsymbol{I}_{\text {diff }}$ maps varied between states (Figure 6B). For example, FPN and DAN showed a larger $-\boldsymbol{\Delta} \boldsymbol{I}_{\text {diff }}$ in State 1 and 2, while DMN showed a larger $-\boldsymbol{\Delta} \boldsymbol{I}_{\text {diff }}$ in State 3 and 4 . In addition, the contribution of each functional network was also estimated by excluding regions that belonged to the network. Similar $-\boldsymbol{\Delta} \boldsymbol{I}_{\text {diff }}$ maps were obtained at the three levels (Figure S5), and networks at the subject-level generally exhibited larger $-\boldsymbol{\Delta} \boldsymbol{I}_{\text {diff }}$ values than the other two levels.

Furthermore, the pattern of variance map and $-\boldsymbol{\Delta} \boldsymbol{I}_{\text {diff }}$ map was compared. A strong positive correlation was found between them, suggesting brain regions with larger variance tend to contribute more to the subject discrimination (Figure 6C). Similar results at the group-level and scan-level were presented in the supplementary materials (Figure S3, S4). 


\section{Subject-Level}

State 1

A

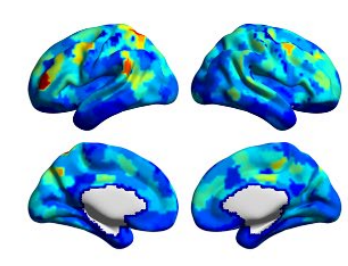

B

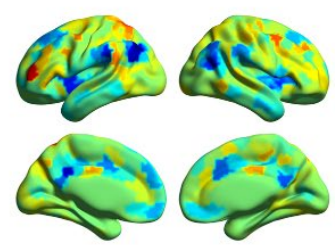

C

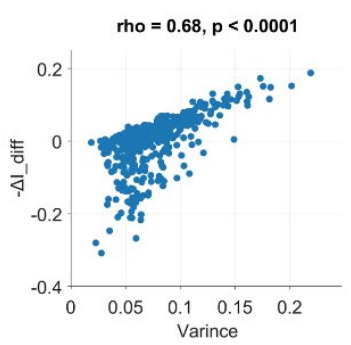

State 2

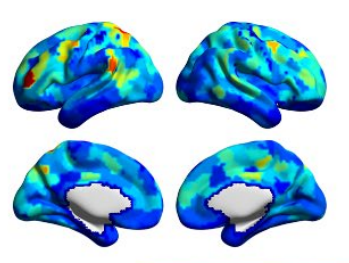

0
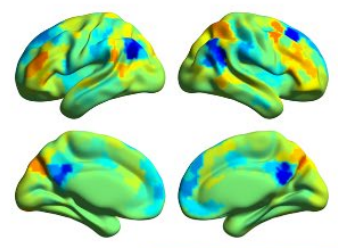

State 3

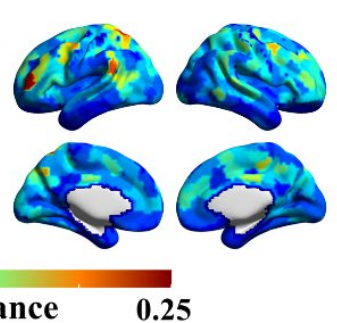

Variance $\quad 0.25$
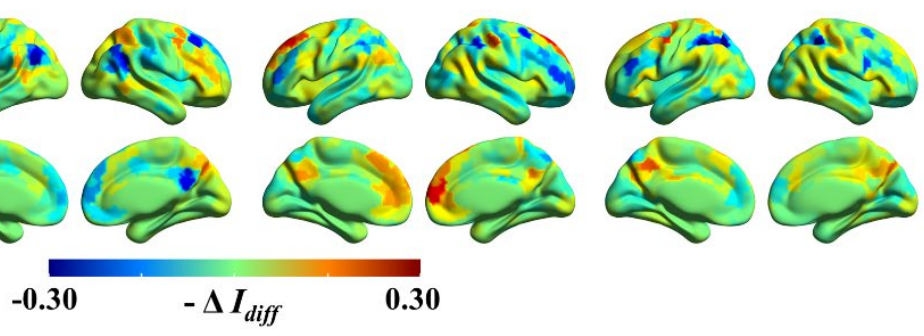

State 4

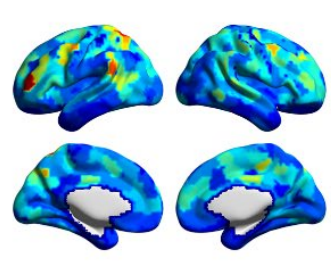

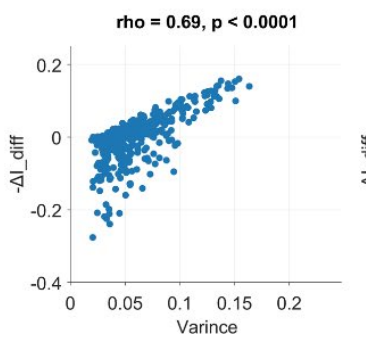
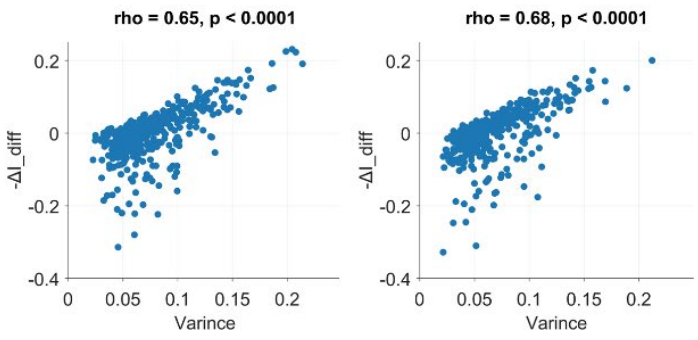

Figure 6. Brain regions contributed to the subject identification at the subject-level. A)

Variance maps of the four CAP states. B) Changed differential identifiability $\left(-\Delta \boldsymbol{I}_{\text {diff }}\right)$ maps of the four CAP states. C) Significant positive correlation was found between the variance map and the $-\boldsymbol{\Delta} \boldsymbol{I}_{\text {diff }}$ map.

\subsection{How does head movement affect CAPs and subject identification}

With visual inspection, it can be observed that several scans showed much less intra-subject similarity than other scans of the same subject, and those scans included more motion-contaminated frames (Figure S8). Therefore, we estimated the effects of the head movement level on intra-subject similarity for each CAP state, by calculating the Spearman correlation between the number of motion-contaminated frames of each 
scan and the $\boldsymbol{I}_{\text {self }}(\boldsymbol{i}, \boldsymbol{j})$ separately. A significant negative correlation was found at each CAP state, suggesting the scan with more motion-contaminated frames was less similar with scans from the same subject (Figure 7A). The scatter plots of State 1 are also presented in Figure 7B as examples. We also compared the ID rate between the highmotion and low-motion scans, while no significant difference (all $\mathrm{P}$ values $>0.05$, uncorrected) was found.

A

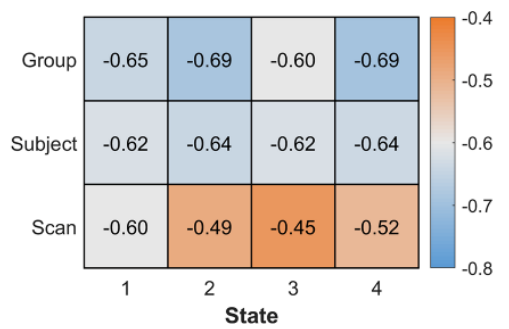

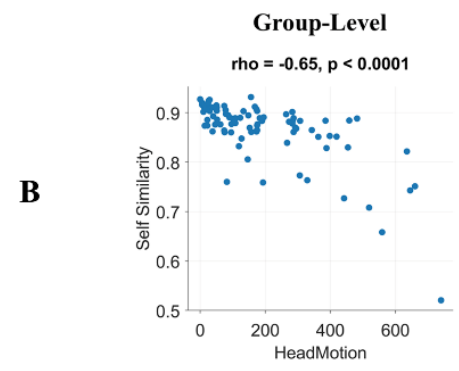

Group-Level

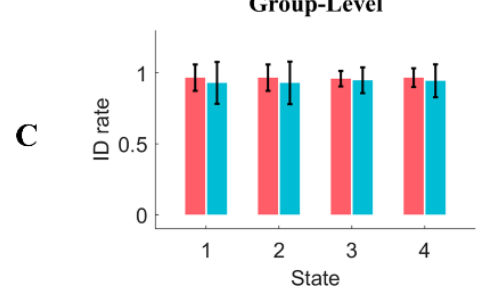

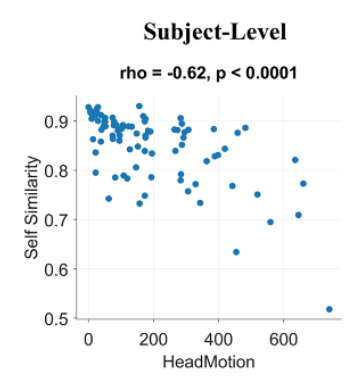

Subject-Level

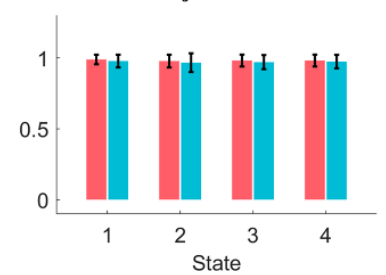

Scan-Level

rho $=-0.60, p<0.0001$

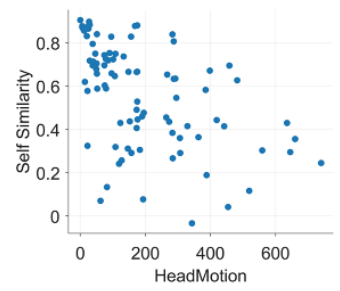

Scan-Level

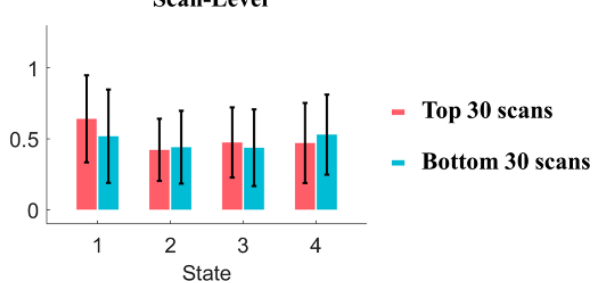

Figure 7. Head movement effects on CAP states. A) The relationship between head motion level and intra-subject similarity. B) Scatter plots for State 1, each point was a scan, the $\mathrm{X}$-axis is the number of motion-contaminated frames, and the Y-axis shows the self-similarity. C) The identification (ID) rate comparisons between the top 30 and bottom 30 scans, ordered by the number of motion-contaminated frames. No significant difference (all $\mathrm{P}$ values $>0.05$, uncorrected) was found by using permutation test. 


\subsection{Associations between CAP dynamics and IQ}

To overcome the limitation of having a minimal sample size of the MSC dataset, we developed a random-based brain-behavior correlation pipeline to augment the sample size (Figure 2). In this study, IQ was used as exploratory research. The true distribution was generated based on the 10,000 augmented data group, and the null distribution was generated by shuffling the correct correspondence between brain measures and IQ. For the three CAP matrices (fraction of time, persistence and counts) at the three levels, the mean correlation values of all null distributions were around zero. Specifically, the results of fraction of time are shown in Figure 8. True distributions at the scan-level were similar to the null distributions, with mean values around zero. However, a large effect size was found at the subject-level. The fraction of time of State 1 showed a positive correlation with IQ $(u=0.25$, Cohen's $d=0.83)$, and a negative association was found in State $3(u=-0.27$, Cohen's $d=-0.82)$. As for the group-level, similar trend but weaker effect size was found in State $1(u=0.15$, Cohen's $d=0.45)$, and medium effect size was found it State $2(u=0.16$, Cohen's $d=0.51)$ and State $4(u$ $=-0.23$, Cohen's $d=-0.74)$. In addition, for persistence, consistent trend was also found in State 1 at the group-level $(u=0.28$, Cohen's $d=0.87)$ and subject-level $(u=0.17$, Cohen's $d=0.57$ ), that the persistence of State 1 was positively correlated with IQ. More detailed results of persistence and counts were described in the supplementary materials (Figure S6, S7). 


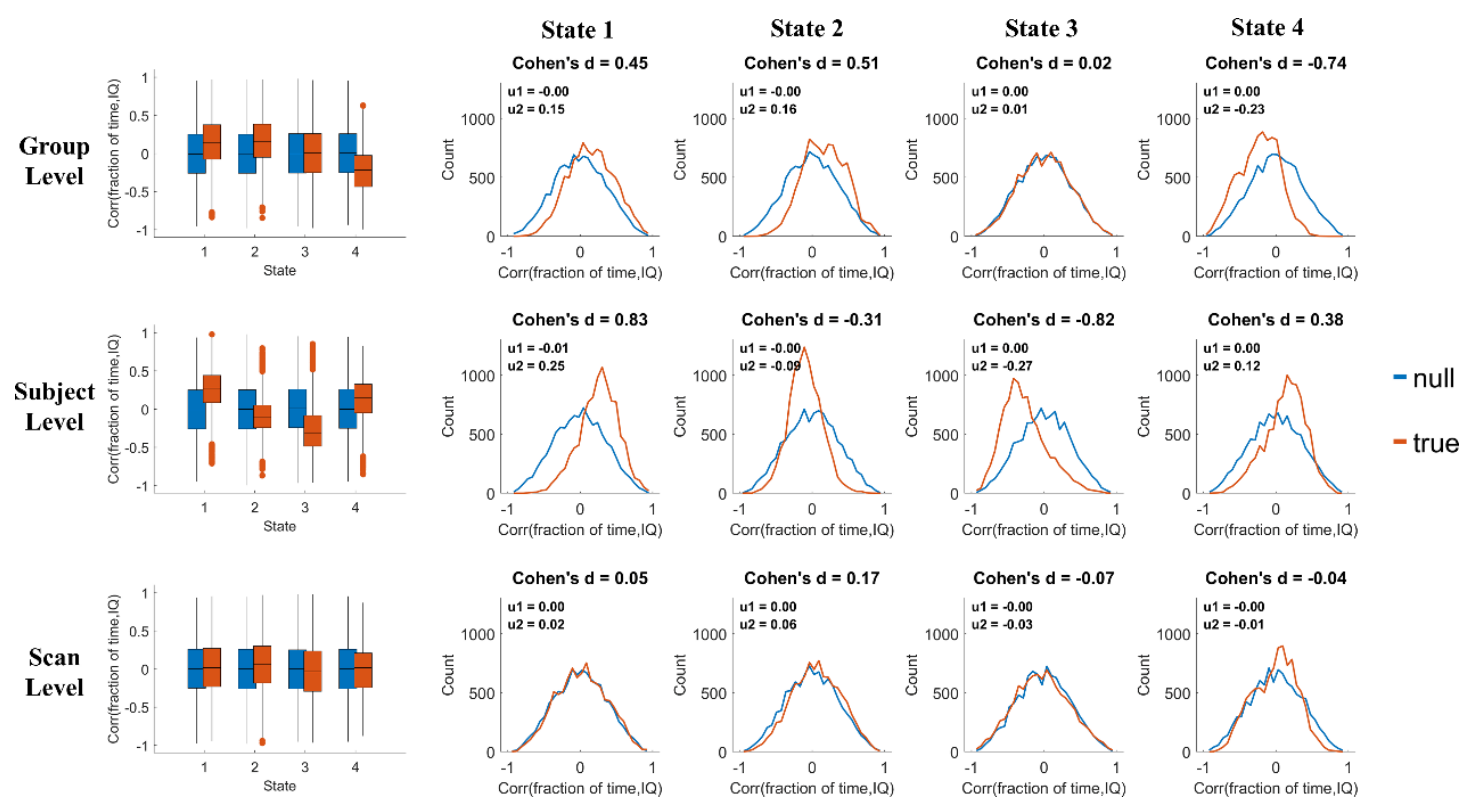

Figure 8. Applications of the brain-behavior correlation pipeline. The true distribution was generated from the augmented data group, and the null distribution was obtained by shuffling the correspondence between brain measure (e.g., fraction of time) and behavior measure (IQ). Cohen's d was calculated to measure the effect size of differences between the true and null distribution, $\mathrm{u} 1$ and $\mathrm{u} 2$ are the mean value of the null distribution and the true distribution, respectively.

\section{Discussion}

The current study identified four CAPs at each of the three levels (group-, subjectand scan-level), and the CAP maps were reconstructed for each session. We first compared the subject identification ability among the three levels. Results of the subject-level achieved the highest identification accuracy and the largest differential identifiability, by enlarging the inter-subject differences while preserving as much intrasubject similarity as possible. Regions at the FPN, DAN and DMN contributed more to the identification. Our results also revealed the effects of head movement on CAPs. 
Finally, we established an analysis pipeline to estimate the brain-behavior relationship in the dataset with a small sample size but multiple repetitions.

\subsection{Subject-level CAPs improved the subject identification}

In this study, the CAPs were generated at the group-level, subject-level and scanlevel. As illustrated in Figures 4 and 5, due to the low intra- and inter-subject similarity, the scan-level failed to identify subjects (ID rate: 0.43 to 0.57 ) and its differential identifiability was low $\left(\boldsymbol{I}_{\text {diff }}<20\right)$. As for the group-level, the high intra- similarity resulted in high identification accuracy $(\sim 0.95)$, while it also exhibited high intersubject similarity (or small between-subject differences), which caused the similar low differential identifiability as the scan-level. However, CAPs at the subject-level not only maintained the high intra-subject similarity but also enhanced the inter-subject differences, hence the highest identification accuracy $(\sim 0.97)$ and largest differential identifiability were obtained. It is known that concatenating multiple scans per subject could improve the reliability of resting-state FC (Cho et al. 2021; Pannunzi et al. 2017). A recent study has also demonstrated that combining multiple scans improved the detection of individual functional modules (Gupta and Rajapakse 2020). These findings indicate that including more data from the individual is helpful for brain studies.

In general, group-level and subject-level have shown a comparable identification rate with previous studies (Finn et al. 2015; Menon and Krishnamurthy 2019). As for the differential identifiability, previous node-FC studies found that $\boldsymbol{I}_{\text {diff }}$ could be optimized by using principal component analysis (PCA) reconstruction (Amico and 
Goni 2018; Bari et al. 2019). With the benefits of higher-order reconstruction, edgecentric FC further improved the subject idiosyncrasies (Jo et al. 2021). In the current study, CAPs at the group-level and scan-level showed similar differential identifiability $\left(\boldsymbol{I}_{\text {diff }}<20\right)$ with previous node-FC results (Amico and Goni 2018; Bari et al. 2019). Notably, the largest $\boldsymbol{I}_{\text {diff }}$ at the subject-level ( $\boldsymbol{I}_{\text {diff }}=36.77$, State 3$)$ was even slightly higher than that of edge-centric FC ( $\boldsymbol{I}_{\text {diff }}=35.27$, PCA optimized) (Jo et al. 2021), suggesting that although coactivation profiles are first-order brain measurement, brain dynamics could provide additional personal traits for subject identification.

\subsection{Discriminative brain regions and networks}

To assess the contribution of brain regions and networks to the subject identification, the changed differential identifiability was calculated by excluding one region or network each time (Jo et al. 2021). The most discriminative brain regions were mainly located in the higher-order networks, such as the FPN, DAN and DMN. These higher-order association cortices are critical for cognition (Cole et al. 2013), the most evolutionarily recent (Sepulcre et al. 2010), and characterized by large individual variability (Laumann et al. 2015; Mueller et al. 2013). Together, these might be the reasons that why these heteromodal regions drive the subject identification (Finn et al. 2015; Horien et al. 2019; Jo et al. 2021). In addition to the changed differential identifiability (- $\left.\boldsymbol{\Delta} \boldsymbol{I}_{\text {diff }}\right)$, the current study also calculated the inter-scan variance of CAPs. Strong positive correlations were found between the variance maps and $-\boldsymbol{\Delta} \boldsymbol{I}_{\text {diff }}$ maps, suggesting that brain regions with larger individual differences tend to be more 
identifiable. Although the two maps were similar, variance maps were almost identical across CAP states, while $-\boldsymbol{\Delta} \boldsymbol{I}_{\text {diff }}$ maps exhibited state-specific profiles. For instance, the FPN and DAN were more identifiable in State 1 and 2, and the DMN was more identifiable in State 3 and 4, indicating the differential identifiability is more sensitive to individual-specific dynamic characteristics.

\subsection{Unstable individual CAP states over time}

Another question is whether stable individual CAP states could be identified in acquisitions over time. Although the scan length of a single session is long enough $(\sim 30$ minutes), consistent CAPs across the ten sessions were only obtained in a few subjects (e.g., MSC02, MSC06), which is different from previous studies that demonstrate individual connectome is stable over time or cognitive states (Gratton et al. 2018; Horien et al. 2019; Seitzman et al. 2019). The reason might be that the previous individual connectome was mainly characterized by using static FC, and as the intrinsic functional architecture, static FC is stable across resting-state and tasks (Cole et al. 2014). However, dynamic FC showed lower reliability than static FC, and highly dynamic connections are less reliable (Zhang et al. 2018). We have shown the CAP states are reproducible across analytic flexibilities and generalizable between independent datasets (Yang et al. 2021b), while the test-retest reliability through multiple scans is remained to be clarified. Besides, one previous study found that the brain state could be manipulated by scan conditions (Finn et al. 2017), and the statespecific individual functional parcellations cannot be obtained using a single 
acquisition (Salehi et al. 2020a). As the underlying unrestricted and unobservable mental process could be varied across resting-state sessions of the same subject, the brain might reconfigure at distinct cognitive states (Krienen et al. 2014; Salehi et al. 2020b) and cause the unstable individual CAP states when using a single scan.

\subsection{The effects of head motion on CAP-related subject identification}

The head motion is a significant nuisance factor analyzed in fMRI studies (Power et al. 2014; Power et al. 2018). It would produce artifacts of the brain structure (Zaitsev et al. 2015), contaminate the BOLD signal, and introduce spurious functional connectivity (Power et al. 2012; Van Dijk et al. 2012). Head motion has shown homogeneous and trait-like properties (Couvy-Duchesne et al. 2014; Siegel et al. 2017), and it also exhibited high test-retest reliability (Zuo and Xing 2014). Furthermore, subject-specific spatiotemporal signatures could be induced by the head movement (Bolton et al. 2020; Xifra-Porxas et al. 2021). These findings indicate that the head motion might contribute to the subject identification. Nevertheless, previous studies reported that, compared with functional connectivity profiles, head motion only achieved a much lower above-chance level in identification rate (Finn et al. 2015; Vanderwal et al. 2017). Consistent with previous findings (Horien et al. 2019), we found that scans with larger head movement showed less inter-scan similarity (Figure 7). Besides, the CAP state with the largest differential identifiability $\left(\boldsymbol{I}_{\text {diff }}=36.77\right.$, State 3$)$ was also less contaminated by head motion (Figure S9), indicating that head motion would reduce the subject identifiability (Amico and Goni 2018). Furthermore, previous 
studies found that low-motion subjects showed a higher identification rate than using all subjects (Horien et al. 2019), and controlling the effects of head motion during the preprocessing increased the accuracy (Xifra-Porxas et al. 2021). The current study did not observe a significant difference in identification rate between low-motion and highmotions scans, suggesting that the differential identifiability might be more sensitive to head motion. In sum, these results support that the subject identification was mainly driven by the neural-origin individual differences rather than head motion.

\subsection{Build brain-behavior relationships in precision fMRI datasets}

At present, there are already several precision fMRI datasets (Gratton et al. 2020), which include a few subjects with dense sampling. The midnight scan club (MSC) is a widely used dataset to explore individual differences (Gordon et al. 2017b), and related studies have mainly focused on subject-specific spatial characteristics, such as individualized parcellations (Greene et al. 2020; Marek et al. 2018), network variants (Kraus et al. 2021; Seitzman et al. 2019) and brain fingerprints (Jo et al. 2021). However, due to the minimal sample size and not enough statistical power to build a reliable brainbehavior relationship, no study has reported results of brain-behavior associations. In this work, we hypothesized that if one brain measure is tightly and reliably related to a specific behavior, then the brain measure estimated by any scan should be appropriate to link with the behavior. Then, based on the idea of resampling and permutation, we manually created a large amount of (e.g., 10,000) augmented groups and shuffled groups, and analyzed their brain-behavior correlations. We believe a certain degree of 
brain-behavior inferences could be made by comparing the distribution of the two groups, as an exploratory analysis.

The previous study has shown that the individual functional connectome could predict an individual's IQ (Finn et al. 2015), and IQ was used in the current study as a preliminary exploration. Overall, no significant difference between the null-distribution and true-distribution was observed at the scan-level, and CAPs at the subject-level showed the strongest correlation (Figure 8). Particularly, for fraction of time at the subject-level, a positive correlation was found in State 1 (mean $r=0.25)$, and a negative correlation was found in State 3 (mean $r=-0.27$ ), suggesting that subjects with higher IQ spent more time in the activated VAN state but less time in the inhibited FPN and DAN state. These high-order networks are related to cognitive control (Cole et al. 2013; Menon and D'Esposito 2022), and associated with individual differences in intelligence (Barbey 2018; Hilger et al. 2017; Santarnecchi et al. 2017). Based on the parieto-frontal integration theory (P-FIT), frontal and parietal cortices are particularly important for intelligence (Jung and Haier 2007; Vakhtin et al. 2014). In addition, the structure of these networks (e.g., grey matter volume, local gyrification, dendritic density and arborization) could also predict individual intelligence (Genc et al. 2018; Gregory et al. 2016; Hilger et al. 2020). Hopefully, this pipeline might be helpful to build brainbehavior associations in the precision dataset with few subjects and dense sampling.

\subsection{Limitations and future directions}

There are several limitations in the current study. Previous static FC-based subject 
identification studies have tested the effect of scan length on subject identification, and they found a longer scan length led to a higher identification accuracy and larger differential identifiability (Amico and Goni 2018; Bari et al. 2019; Jo et al. 2021). The effects of scan length were not measured in this study, as enough time points are required to obtain robust CAPs and estimate their temporal dynamics (Liu et al. 2018). Nevertheless, a recent magnetoencephalography study suggested that even a short brain recording ( $\sim 30$ seconds) could also achieve differentiation (da Silva Castanheira et al. 2021). In the current study, the effects of head motion on CAPs and subject identification have been studied by evaluating how it affects the intra-subject similarity and comparing the ID rate between the high-motion and low-motion groups. However, no further contrast experiment was performed as we used the preprocessed fMRI images from the open dataset. Future studies should take different head motion control strategies during the preprocessing into account. Last but not the least, rather than using the group-level atlas, personalized parcellations should also be considered to evaluate the individual CAPs.

\section{Conclusion}

The current study has shown that the subject-level CAPs improved the subject identification, and heteromodal regions from the higher-order networks (e.g., FPN, DAN, DMN) exhibited more individual idiosyncrasies. Besides, stable individual CAPs states were not obtained when using a single resting-state scan, and head motion would reduce the intra-subject similarity. Finally, by developing a random-based pipeline, we 
tried to uncover potential brain-behavior inferences in datasets with few subjects but multiple scans.

\section{Data and code availability}

The preprocessed Midnight Scan Club (MSC) data used in this study are available in the OpenfMRI data repository at https://openneuro.org/datasets/ds000224.

The codes for CAP generation and CAP matrices calculation can be found in https://github.com/davidyoung1994/CoactivationPattern.

\section{Funding}

This work was supported by the National Natural Science Foundation of China (NSFC) grant (No. 61871420, 62171101 to B.B.).

\section{Conflict of interest}

The authors declare no conflict of interest.

\section{Ethics approval and patient consent}

The study was approved by the Washington University School of Medicine Human

Studies Committee and Institutional Review Board. The written informed consent was obtained from all participants.

\section{Acknowledgements}


We thank Evan M. Gordon and his team for collecting and sharing the midnight scan club data. This work was supported by the National Natural Science Foundation of China (NSFC) grant (No. 62071109 to C.M., No. 61871420 to B.B.,).

\section{References}

Allen EA, Damaraju E, Plis SM, Erhardt EB, Eichele T, Calhoun VD. 2014. Tracking whole-brain connectivity dynamics in the resting state. Cerebral Cortex. 24(3):663-676.

Amico E, Goni J. 2018. The quest for identifiability in human functional connectomes. Sci Rep. $8(1): 8254$.

Barbey AK. 2018. Network neuroscience theory of human intelligence. Trends Cogn Sci. 22(1):8-20.

Bari S, Amico E, Vike N, Talavage TM, Goni J. 2019. Uncovering multi-site identifiability based on resting-state functional connectomes. Neuroimage. 202:115967.

Bolton TAW, Kebets V, Glerean E, Zoller D, Li JW, Yeo BTT, Caballero-Gaudes C, Van De Ville D. 2020. Agito ergo sum: Correlates of spatio-temporal motion characteristics during fmri. Neuroimage. 209.

Buckner RL, Krienen FM, Yeo BT. 2013. Opportunities and limitations of intrinsic functional connectivity mri. Nat Neurosci. 16(7):832-837.

Cash RFH, Cocchi L, Lv J, Wu Y, Fitzgerald PB, Zalesky A. 2021. Personalized connectivity-guided dlpfc-tms for depression: Advancing computational feasibility, precision and reproducibility. Hum Brain Mapp. 42(13):4155-4172.

Chen JE, Chang C, Greicius MD, Glover GH. 2015. Introducing co-activation pattern metrics to quantify spontaneous brain network dynamics. Neuroimage. 111:476-488.

Cho JW, Korchmaros A, Vogelstein JT, Milham MP, Xu T. 2021. Impact of concatenating fmri data on reliability for functional connectomics. Neuroimage. 226.

Cohen AD, Chang C, Wang Y. 2021. Using multiband multi-echo imaging to improve the robustness and repeatability of co-activation pattern analysis for dynamic functional connectivity. Neuroimage. 243:118555.

Cole MW, Bassett DS, Power JD, Braver TS, Petersen SE. 2014. Intrinsic and task-evoked network architectures of the human brain. Neuron. 83(1):238-251.

Cole MW, Reynolds JR, Power JD, Repovs G, Anticevic A, Braver TS. 2013. Multi-task connectivity reveals flexible hubs for adaptive task control. Nature Neuroscience. 16(9):1348-U1247.

Couvy-Duchesne B, Blokland GA, Hickie IB, Thompson PM, Martin NG, de Zubicaray GI, McMahon KL, Wright MJ. 2014. Heritability of head motion during resting state functional mri in 462 healthy twins. Neuroimage. 102 Pt 2:424-434.

Cui ZX, Li HM, Xia CH, Larsen B, Adebimpe A, Baum GL, Cieslak M, Gur RE, Gur RC, Moore TM et al. 2020. Individual variation in functional topography of association networks in youth. Neuron. 106(2):340-+.

da Silva Castanheira J, Orozco Perez HD, Misic B, Baillet S. 2021. Brief segments of neurophysiological activity enable individual differentiation. Nat Commun. 12(1):5713. 
Dubois J, Adolphs R. 2016. Building a science of individual differences from fmri. Trends Cogn Sci. 20(6):425-443.

Finn ES, Scheinost D, Finn DM, Shen XL, Papademetris X, Constable RT. 2017. Can brain state be manipulated to emphasize individual differences in functional connectivity? Neuroimage. 160:140-151.

Finn ES, Shen XL, Scheinost D, Rosenberg MD, Huang J, Chun MM, Papademetris X, Constable RT. 2015. Functional connectome fingerprinting: Identifying individuals using patterns of brain connectivity. Nature Neuroscience. 18(11):1664-1671.

Fornito A, Zalesky A, Breakspear M. 2015. The connectomics of brain disorders. Nat Rev Neurosci. 16(3):159-172.

Genc E, Fraenz C, Schluter C, Friedrich P, Hossiep R, Voelkle MC, Ling JM, Gunturkun O, Jung RE. 2018. Diffusion markers of dendritic density and arborization in gray matter predict differences in intelligence. Nat Commun. 9(1):1905.

Glasser MF, Coalson TS, Robinson EC, Hacker CD, Harwell J, Yacoub E, Ugurbil K, Andersson J, Beckmann CF, Jenkinson M et al. 2016. A multi-modal parcellation of human cerebral cortex. Nature. 536(7615):171-178.

Gordon EM, Laumann TO, Adeyemo B, Gilmore AW, Nelson SM, Dosenbach NUF, Petersen SE. 2017a. Individual-specific features of brain systems identified with resting state functional correlations. Neuroimage. 146:918-939.

Gordon EM, Laumann TO, Gilmore AW, Newbold DJ, Greene DJ, Berg JJ, Ortega M, Hoyt-Drazen C, Gratton C, Sun $\mathrm{H}$ et al. 2017b. Precision functional mapping of individual human brains. Neuron. 95(4):791-807 e797.

Gratton C, Kraus BT, Greene DJ, Gordon EM, Laumann TO, Nelson SM, Dosenbach NUF, Petersen SE. 2020. Defining individual-specific functional neuroanatomy for precision psychiatry. Biol Psychiatry. 88(1):28-39.

Gratton C, Laumann TO, Nielsen AN, Greene DJ, Gordon EM, Gilmore AW, Nelson SM, Coalson RS, Snyder AZ, Schlaggar BL et al. 2018. Functional brain networks are dominated by stable group and individual factors, not cognitive or daily variation. Neuron. 98(2):439-452 e435.

Greene DJ, Marek S, Gordon EM, Siegel JS, Gratton C, Laumann TO, Gilmore AW, Berg JJ, Nguyen $\mathrm{AL}$, Dierker D et al. 2020. Integrative and network-specific connectivity of the basal ganglia and thalamus defined in individuals. Neuron. 105(4):742-758 e746.

Gregory MD, Kippenhan JS, Dickinson D, Carrasco J, Mattay VS, Weinberger DR, Berman KF. 2016. Regional variations in brain gyrification are associated with general cognitive ability in humans. Curr Biol. 26(10):1301-1305.

Gupta S, Rajapakse JC. 2020. Iterative consensus spectral clustering improves detection of subject and group level brain functional modules. Sci Rep. 10(1):7590.

Gutierrez-Barragan D, Basson MA, Panzeri S, Gozzi A. 2019. Infraslow state fluctuations govern spontaneous fmri network dynamics. Curr Biol. 29(14):2295-2306 e2295.

Hilger K, Ekman M, Fiebach CJ, Basten U. 2017. Efficient hubs in the intelligent brain: Nodal efficiency of hub regions in the salience network is associated with general intelligence. Intelligence. 60:10-25.

Hilger K, Winter NR, Leenings R, Sassenhagen J, Hahn T, Basten U, Fiebach CJ. 2020. Predicting intelligence from brain gray matter volume. Brain Struct Funct. 225(7):2111-2129.

Horien C, Shen XL, Scheinost D, Constable RT. 2019. The individual functional connectome is unique 
and stable over months to years. Neuroimage. 189:676-687.

Huang Z, Zhang J, Wu J, Mashour GA, Hudetz AG. 2020. Temporal circuit of macroscale dynamic brain activity supports human consciousness. Sci Adv. 6(11):eaaz0087.

Hutchison RM, Womelsdorf T, Allen EA, Bandettini PA, Calhoun VD, Corbetta M, Penna S, Duyn JH, Glover GH, Gonzalez-Castillo J et al. 2013. Dynamic functional connectivity: Promise, issues, and interpretations. Neuroimage. 80:360-378.

Jo Y, Faskowitz J, Esfahlani FZ, Sporns O, Betzel RF. 2021. Subject identification using edge-centric functional connectivity. Neuroimage. 238.

Jung RE, Haier RJ. 2007. The parieto-frontal integration theory (p-fit) of intelligence: Converging neuroimaging evidence. Behav Brain Sci. 30(2):135-154; discussion 154-187.

Kaiser RH, Kang MS, Lew Y, Van Der Feen J, Aguirre B, Clegg R, Goer F, Esposito E, Auerbach RP, Hutchison RM et al. 2019. Abnormal frontoinsular-default network dynamics in adolescent depression and rumination: A preliminary resting-state co-activation pattern analysis. Neuropsychopharmacology. 44(9):1604-1612.

Kaufman ASJCP, MN: American Guidance Service. 2004. Kaufman brief intelligence test-second edition (kbit-2).

Kaufmann T, Alnaes D, Doan NT, Brandt CL, Andreassen OA, Westlye LT. 2017. Delayed stabilization and individualization in connectome development are related to psychiatric disorders. Nature Neuroscience. 20(4):513-+.

Kong R, Li J, Orban C, Sabuncu MR, Liu H, Schaefer A, Sun N, Zuo XN, Holmes AJ, Eickhoff SB et al. 2019. Spatial topography of individual-specific cortical networks predicts human cognition, personality, and emotion. Cereb Cortex. 29(6):2533-2551.

Kraus BT, Perez D, Ladwig Z, Seitzman BA, Dworetsky A, Petersen SE, Gratton C. 2021. Network variants are similar between task and rest states. Neuroimage. 229.

Krienen FM, Yeo BT, Buckner RL. 2014. Reconfigurable task-dependent functional coupling modes cluster around a core functional architecture. Philos Trans R Soc Lond B Biol Sci. 369(1653).

Kuhn HW. 1955. The hungarian method for the assignment problem. 2(1-2):83-97.

Kumar K, Desrosiers C, Siddiqi K, Colliot O, Toews M. 2017. Fiberprint: A subject fingerprint based on sparse code pooling for white matter fiber analysis. Neuroimage. 158:242-259.

Kupis L, Goodman ZT, Kornfeld S, Hoang S, Romero C, Dirks B, Dehoney J, Chang C, Spreng RN, Nomi JS et al. 2021. Brain dynamics underlying cognitive flexibility across the lifespan. Cereb Cortex. 31(11):5263-5274.

Laumann TO, Gordon EM, Adeyemo B, Snyder AZ, Joo SJ, Chen MY, Gilmore AW, McDermott KB, Nelson SM, Dosenbach NU et al. 2015. Functional system and areal organization of a highly sampled individual human brain. Neuron. 87(3):657-670.

Liu X, Chang C, Duyn JH. 2013. Decomposition of spontaneous brain activity into distinct fmri coactivation patterns. Front Syst Neurosci. 7:101.

Liu X, Duyn JH. 2013. Time-varying functional network information extracted from brief instances of spontaneous brain activity. Proc Natl Acad Sci U S A. 110(11):4392-4397.

Liu X, Zhang N, Chang C, Duyn JH. 2018. Co-activation patterns in resting-state fmri signals. Neuroimage. 180(Pt B):485-494.

Lynch CJ, Breeden AL, Gordon EM, Cherry JBC, Turkeltaub PE, Vaidya CJ. 2019. Precision inhibitory stimulation of individual-specific cortical hubs disrupts information processing in humans. Cereb Cortex. 29(9):3912-3921. 
Marek S, Siegel JS, Gordon EM, Raut RV, Gratton C, Newbold DJ, Ortega M, Laumann TO, Adeyemo B, Miller DB et al. 2018. Spatial and temporal organization of the individual human cerebellum. Neuron. 100(4):977-993 e977.

Marquand AF, Rezek I, Buitelaar J, Beckmann CF. 2016. Understanding heterogeneity in clinical cohorts using normative models: Beyond case-control studies. Biol Psychiat. 80(7):552-561.

Menon SS, Krishnamurthy K. 2019. A comparison of static and dynamic functional connectivities for identifying subjects and biological sex using intrinsic individual brain connectivity. Sci Rep-Uk. 9.

Menon V, D'Esposito M. 2022. The role of pfc networks in cognitive control and executive function. Neuropsychopharmacology. 47(1):90-103.

Mueller S, Wang D, Fox MD, Yeo BT, Sepulcre J, Sabuncu MR, Shafee R, Lu J, Liu H. 2013. Individual variability in functional connectivity architecture of the human brain. Neuron. 77(3):586-595.

Murray L, Maurer JM, Peechatka AL, Frederick BB, Kaiser RH, Janes AC. 2021. Sex differences in functional network dynamics observed using coactivation pattern analysis. Cogn Neurosci. 12(3-4):120-130.

Pallares V, Insabato A, Sanjuan A, Kuhn S, Mantini D, Deco G, Gilson M. 2018. Extracting orthogonal subject- and condition-specific signatures from fmri data using whole-brain effective connectivity. Neuroimage. 178:238-254.

Pannunzi M, Hindriks R, Bettinardi RG, Wenger E, Lisofsky N, Martensson J, Butler O, Filevich E, Becker M, Lochstet M et al. 2017. Resting-state fmri correlations: From link-wise unreliability to whole brain stability. Neuroimage. 157:250-262.

Piguet C, Karahanoglu FI, Saccaro LF, Van De Ville D, Vuilleumier P. 2021. Mood disorders disrupt the functional dynamics, not spatial organization of brain resting state networks. Neuroimage Clin. 32:102833.

Power JD, Barnes KA, Snyder AZ, Schlaggar BL, Petersen SE. 2012. Spurious but systematic correlations in functional connectivity mri networks arise from subject motion. Neuroimage. 59(3):2142-2154.

Power JD, Mitra A, Laumann TO, Snyder AZ, Schlaggar BL, Petersen SE. 2014. Methods to detect, characterize, and remove motion artifact in resting state fmri. Neuroimage. 84:320-341.

Power JD, Plitt M, Gotts SJ, Kundu P, Voon V, Bandettini PA, Martin A. 2018. Ridding fmri data of motion-related influences: Removal of signals with distinct spatial and physical bases in multiecho data. Proc Natl Acad Sci U S A. 115(9):E2105-E2114.

Preti MG, Bolton TAW, Van De Ville D. 2017. The dynamic functional connectome: State-of-the-art and perspectives. Neuroimage. 160:41-54.

Price RB, Lane S, Gates K, Kraynak TE, Horner MS, Thase ME, Siegle GJ. 2017. Parsing heterogeneity in the brain connectivity of depressed and healthy adults during positive mood. Biol Psychiat. 81(4):347-357.

Rey G, Bolton TAW, Gaviria J, Piguet C, Preti MG, Favre S, Aubry JM, Van De Ville D, Vuilleumier P. 2021. Dynamics of amygdala connectivity in bipolar disorders: A longitudinal study across mood states. Neuropsychopharmacology. 46(9):1693-1701.

Salehi M, Greene AS, Karbasi A, Shen XL, Scheinost D, Constable RT. 2020a. There is no single functional atlas even for a single individual: Functional parcel definitions change with task. Neuroimage. 208.

Salehi M, Karbasi A, Barron DS, Scheinost D, Constable RT. 2020b. Individualized functional networks 
reconfigure with cognitive state. Neuroimage. 206:116233.

Santarnecchi E, Emmendorfer A, Pascual-Leone A, Authors HST. 2017. Dissecting the parieto-frontal correlates of fluid intelligence: A comprehensive ale meta-analysis study. Intelligence. 63:9-28.

Schaefer A, Kong R, Gordon EM, Laumann TO, Zuo XN, Holmes AJ, Eickhoff SB, Yeo BTT. 2018. Local-global parcellation of the human cerebral cortex from intrinsic functional connectivity mri. Cereb Cortex. 28(9):3095-3114.

Seitzman BA, Gratton C, Laumann TO, Gordon EM, Adeyemo B, Dworetsky A, Kraus BT, Gilmore AW, Berg JJ, Ortega $\mathrm{M}$ et al. 2019. Trait-like variants in human functional brain networks. Proc Natl Acad Sci U S A. 116(45):22851-22861.

Sepulcre J, Liu H, Talukdar T, Martincorena I, Yeo BT, Buckner RL. 2010. The organization of local and distant functional connectivity in the human brain. PLoS Comput Biol. 6(6):e1000808.

Siegel JS, Mitra A, Laumann TO, Seitzman BA, Raichle M, Corbetta M, Snyder AZ. 2017. Data quality influences observed links between functional connectivity and behavior. Cerebral Cortex. 27(9):4492-4502.

Sorrentino P, Rucco R, Lardone A, Liparoti M, Lopez ET, Cavaliere C, Soricelli A, Jirsa V, Sorrentino G, Amico E. 2021. Clinical connectome fingerprints of cognitive decline. Neuroimage. 238.

Sui J, Jiang R, Bustillo J, Calhoun V. 2020. Neuroimaging-based individualized prediction of cognition and behavior for mental disorders and health: Methods and promises. Biol Psychiatry. 88(11):818-828.

Sylvester CM, Yu Q, Srivastava AB, Marek S, Zheng A, Alexopoulos D, Smyser CD, Shimony JS, Ortega M, Dierker DL et al. 2020. Individual-specific functional connectivity of the amygdala: A substrate for precision psychiatry. Proc Natl Acad Sci U S A. 117(7):3808-3818.

Tarun A, Behjat H, Bolton T, Abramian D, Van De Ville D. 2020. Structural mediation of human brain activity revealed by white-matter interpolation of fmri. Neuroimage. 213:116718.

Vakhtin AA, Ryman SG, Flores RA, Jung RE. 2014. Functional brain networks contributing to the parieto-frontal integration theory of intelligence. Neuroimage. 103:349-354.

Van Dijk KR, Sabuncu MR, Buckner RL. 2012. The influence of head motion on intrinsic functional connectivity mri. Neuroimage. 59(1):431-438.

Vanderwal T, Eilbott J, Finn ES, Craddock RC, Turnbull A, Castellanos FX. 2017. Individual differences in functional connectivity during naturalistic viewing conditions. Neuroimage. 157:521-530.

Vidaurre D, Smith SM, Woolrich MW. 2017. Brain network dynamics are hierarchically organized in time. Proc Natl Acad Sci U S A. 114(48):12827-12832.

Wang D, Buckner RL, Fox MD, Holt DJ, Holmes AJ, Stoecklein S, Langs G, Pan R, Qian T, Li K et al. 2015. Parcellating cortical functional networks in individuals. Nat Neurosci. 18(12):1853-1860.

Wang D, Tian Y, Li M, Dahmani L, Wei Q, Bai T, Galie F, Ren J, Farooq RK, Wang K et al. 2020. Functional connectivity underpinnings of electroconvulsive therapy-induced memory impairments in patients with depression. Neuropsychopharmacology. 45(9):1579-1587.

Wang DH, Li ML, Wang MY, Schoeppe F, Ren JX, Chen HF, ?ng?r D, Brady RO, Baker JT, Liu HS. 2018. Individual-specific functional connectivity markers track dimensional and categorical features of psychotic illness (vol 25, 2119, 2020). Mol Psychiatr. 25(9):2200-2200.

Xifra-Porxas A, Kassinopoulos M, Mitsis GD. 2021. Physiological and motion signatures in static and time-varying functional connectivity and their subject identifiability. Elife. 10.

Yang H, Zhang H, Di X, Wang S, Meng C, Tian L, Biswal B. 2021a. Frequency-specific coactivation patterns in resting-state and their alterations in schizophrenia: An fmri study. 
Yang H, Zhang H, Di X, Wang S, Meng C, Tian L, Biswal B. 2021b. Reproducible coactivation patterns of functional brain networks reveal the aberrant dynamic state transition in schizophrenia. Neuroimage. 118193.

Yeo BT, Krienen FM, Sepulcre J, Sabuncu MR, Lashkari D, Hollinshead M, Roffman JL, Smoller JW, Zollei L, Polimeni JR et al. 2011. The organization of the human cerebral cortex estimated by intrinsic functional connectivity. J Neurophysiol. 106(3):1125-1165.

Zhang C, Baum SA, Adduru VR, Biswal BB, Michael AM. 2018. Test-retest reliability of dynamic functional connectivity in resting state fmri. Neuroimage. 183:907-918.

Zuo XN, Xing XX. 2014. Test-retest reliabilities of resting-state fmri measurements in human brain functional connectomics: A systems neuroscience perspective. Neurosci Biobehav Rev. 45:100118 . 